Bundesgesundheitsbl 2014 · 57:762-770

DOI 10.1007/s00103-014-1974-8

c) Springer-Verlag Berlin Heidelberg 2014
T. Lampert · S. Müters · H. Stolzenberg · L. E. Kroll · KiGGS Study Group

Abteilung für Epidemiologie und Gesundheitsmonitoring, Robert Koch-Institut, Berlin

\title{
Messung des sozioökonomischen Status in der KiGGS-Studie
}

\section{Erste Folgebefragung (KiGGS Welle 1)}

Eine Vielzahl nationaler und internationaler Studien weist darauf hin, dass der sozioökonomische Status der Familie einen erheblichen Einfluss auf die gesundheitliche Entwicklung von Kindern und Jugendlichen hat [1-6]. Mit den Daten der Basiserhebung der Studie zur Gesundheit von Kindern und Jugendlichen in Deutschland (KiGGS-Basiserhebung), die in den Jahren 2003 bis 2006 erhoben wurden, konnte beispielsweise gezeigt werden, dass Kinder und Jugendliche aus sozioökonomisch benachteiligten Elternhäusern häufiger einen schlechten allgemeinen Gesundheitszustand aufweisen, vermehrt psychische und Verhaltensauffälligkeiten zeigen sowie zu einem größeren Anteil keinen Sport treiben, sich ungesund ernähren und übergewichtig oder sogar adipös sind [7-9].

Der sozioökonomische Status wurde in der KiGGS-Basiserhebung anhand eines Index erfasst, der auf Angaben der Eltern zu ihrem Bildungsniveau, zu ihrer beruflichen Stellung sowie zum Haushaltsnettoeinkommen basiert [10, 11]. Als Referenz diente dabei ein auf Winkler und Stolzenberg zurückgehender Operationalisierungsvorschlag, der ursprünglich für den Bundes-Gesundheitssurvey 1998 entwickelt wurde und auch in den im Zeitraum 2003 bis 2006 durchgeführten telefonischen Gesundheitssurveys des Robert Koch-Instituts (RKI) in vergleichbarer Weise ungesetzt wurde (WSI-Schichtindex) [12].

In den letzten Jahren wurde am RKI ein Gesundheitsmonitoring etabliert, das künftig eine regelmäßige Verfügbarkeit von Daten zur gesundheitlichen Situation von Kindern, Jugendlichen und Erwach- senen gewährleisten und Aussagen über gesundheitspolitisch relevante zeitliche Entwicklungen und Trends ermöglichen wird [13]. In diesem Zusammenhang wurde die Operationalisierung des sozioökonomischen Status einer kritischen Überprüfung unterzogen und unter Berücksichtigung der Herausforderungen, die sich an das Gesundheitsmonitoring stellen, weiterentwickelt. Neben der Analyse zeitlicher Entwicklungen und Trends sind als Herausforderungen insbesondere die internationale Vergleichbarkeit der Daten sowie der Politik- und Praxistransfer der Ergebnisse zu nennen. Die Umsetzung der überarbeiteten Operationalisierung erfolgte zunächst in den auf Erwachsene bezogenen Komponenten des Gesundheitsmonitorings, d. h. der Studie „Gesundheit in Deutschland aktuell“ (GEDA) [14] und der „Studie zur Gesundheit Erwachsener in Deutschland“ (DEGS1) [15]. Für die Folgebefragung der Studie zur Gesundheit von Kindern und Jugendlichen in Deutschland (KiGGS Welle 1) wird nun eine entsprechende Anpassung vorgenommen und damit eine einheitliche Operationalisierung des sozioökonomischen Status in allen 3 Komponenten des Gesundheitsmonitorings sichergestellt.

Ziel dieses Beitrages ist es, die für KiGGS Welle 1 überarbeitete Operationalisierung des sozioökonomischen Status detailliert zu beschreiben. Dabei wird zunächst darauf eingegangen, wie die einbezogenen Variablen erhoben und wie sie für die Indexbildung aufbereitet wurden. Außerdem werden die Berechnung des Index des sozioökonomischen Status (SES) und die Abgrenzung der sozioökonomischen Statusgruppen dargestellt.
Anschließend werden exemplarisch Ergebnisse zum Zusammenhang zwischen dem sozioökonomischen Status und dem allgemeinen Gesundheitszustand der Kinder und Jugendlichen berichtet, wobei neben dem SES-Index auch eine Betrachtung anhand der Einzelindikatoren erfolgt, die Aussagen über die relative Bedeutung der Bildung und der beruflichen Stellung der Eltern sowie der Einkommenssituation des Haushaltes ermöglicht. In der abschließenden Diskussion werden die Änderungen gegenüber der bisherigen Operationalisierung und die daraus resultierenden Vorteile erörtert sowie die Aussagekraft und die Verwendungsmöglichkeiten des SES-Index beurteilt.

\section{Datengrundlage und statistische Analyse}

KiGGS ist Bestandteil des Gesundheitsmonitorings des RKI und derzeit als kombinierte Querschnitt- und Kohortenstudie realisiert. Ziele, Konzept und Design von KiGGS sind an anderer Stelle ausführlich beschrieben [16-18]. Für den Altersbereich 0 bis 17 Jahre soll KiGGS wiederholt bundesweit erhobene Prävalenzdaten zur gesundheitlichen Situation der in Deutschland lebenden Kinder und Jugendlichen liefern. Die KiGGSBasiserhebung (2003-2006) umfasste Befragungen, Untersuchungen und Laboranalysen, KiGGS Welle 1 (2009-2012) Befragungen in Form von Telefoninterviews. An der KiGGS-Basiserhebung

Die KiGGS Study Group: Die Abteilung Epidemiologie und Gesundheitsmonitoring im Robert Koch-Institut. 
war eine Querschnittstichprobe von insgesamt 17.641 Probanden im Alter von 0 bis 17 Jahren bei einer Response von $66,6 \%$ beteiligt. Die Einzuladenden wurden in einer geschichteten Zufallsstichprobe von 167 Orten Deutschlands zufällig aus den Melderegistern gezogen [16]. Die Stichprobe von KiGGS Welle 1 bestand zum einen aus einer neuen Querschnittstichprobe 0- bis 6-Jähriger, die wiederum zufällig aus den Melderegistern der ursprünglichen 167 Studienorte gezogen wurden. Zum anderen wurden die ehemaligen Teilnehmenden der KiGGS-Basiserhebung, die inzwischen 6 bis 24 Jahre alt waren und als geschlossene Kohorte weitergeführt werden, zur Befragung eingeladen. Die Telefoninterviews wurden durch geschultes Studienpersonal im RKI durchgeführt. Zum Anrufmanagement und zur Datenerfassung wurde das Softwareprodukt Voxco Version 5.4.4.5 (Voxco Inc., Montréal QC, Kanada) eingesetzt. Vor Beginn der Studie lagen positive Voten der Ethikkommission der Charité-Universitätsmedizin Berlin und des Bundesbeauftragten für den Datenschutz vor, eine Befragung erfolgte nur nach Information und schriftlicher Einverständniserklärung der Sorgeberechtigten Minderjähriger oder der volljährigen Probanden selbst. Insgesamt nahmen 12.368 Kinder und Jugendliche (6093 Mädchen, 6275 Jungen) in dem für den Querschnitt relevanten Altersbereich von 0 bis 17 Jahren teil, darunter 4455 Ersteingeladene (Response 38,8\%) und 7913 Wiedereingeladene (Response 72,9\%).

Für die Operationalisierung des sozioökonomischen Status wurden Angaben der Eltern zu ihrer Schulbildung und beruflichen Qualifikation, zu ihrer beruflichen Stellung und beruflichen Tätigkeit, zu ihrem Erwerbsstatus, zum Haushaltsnettoeinkommen, zur Haushaltszusammensetzung und zum Wohnort berücksichtigt.

Die Analysen zum Zusammenhang zwischen dem sozioökonomischen Status und dem allgemeinen Gesundheitszustand der Kinder und Jugendlichen basieren auf der Einschätzung der Eltern zum Gesundheitszustand ihrer Kinder, da die Selbsteinschätzung nur von den 11bis 17-Jährigen erhoben wurde. Die Eltern wurden entsprechend einer von der Weltgesundheitsorganisation empfohlenen Formulierung [19] gefragt: „Wie würden Sie den Gesundheitszustand Ihres Kindes im Allgemeinen beschreiben?" (Antwortkategorien: „sehr gut“, „gut“, „mittelmäßig“, „schlecht“, ,sehr schlecht“). Einbezogen wurden alle Kinder und Jugendlichen im Alter von 3 bis 17 Jahren $(n=11.014)$.

Neben Prävalenzen werden Odds Ratios (OR) für einen mittelmäßigen bis sehr schlechten allgemeinen Gesundheitszustand berichtet. Die Odds Ratios wurden mit binär logistischen Regressionsanalysen berechnet. Sie sind als Chancenverhältnisse $\mathrm{zu}$ interpretieren und bringen zum Ausdruck, um welchen Faktor die Chance für einen mittelmäßigen bis sehr schlechten allgemeinen Gesundheitszustand in der niedrigen bzw. mittleren gegenüber der hohen Statusgruppe, die als Referenzkategorie definiert wurde, erhöht ist. Um nicht von der Chance auf einen schlechten allgemeinen Gesundheitszustand sprechen zu müssen, wird der Begriff des Risikos verwendet und damit eine begriffliche Ungenauigkeit in Kauf genommen.

Zum Vergleich der relativen Bedeutung der 3 Einzelindikatoren des SES für den allgemeinen Gesundheitszustand wird der Relative Index auf Inequality (RII) verwendet [20, 21]. Es handelt sich um eine international gebräuchliche, modellbasiert geschätzte Maßzahl für das Ausmaß gesundheitlicher Ungleichheiten [22]. Odds Ratios sind der allgemeine Standard für die Analyse des Ausmaßes gesundheitlicher Ungleichheit, haben aber den Nachteil, dass sie durch die Wahl der Grenzwerte und die Zellbesetzung der verglichenen Kategorien beeinflusst werden. Der RII hingegen wird nicht durch unterschiedliche Zellbesetzungen in den Teildimensionen beeinflusst. Er eignet sich dadurch besonders für den Vergleich der relativen Bedeutung der Einzelindikatoren des SES, die unterschiedlich differenziert sind und sich nicht in Variablen mit der gleichen Anzahl von gleich stark besetzten Kategorien (beispielsweise Quintile) rekodieren lassen. Der RII beschreibt die Differenz im allgemeinen Gesundheitszustand zwischen der Person mit dem - gemessen am jeweiligen SES-Indikator - niedrigsten und höchs- ten Status. Die Berechnung erfolgt im Fall von abhängigen Merkmalen mit mehr als 2 Kategorien - wie dem fünfstufig erfassten allgemeinen Gesundheitszustand anhand Regressionsmodellen für Zähldaten (Poisson-Regressionsmodelle), die den gesamten Variationsbereich des Outcomes berücksichtigen. Der SES-Indikator wird dazu auf den Variationsbereich 0 bis 1 rekodiert. Die neuen Werte spiegeln jeweils den Anteil der Fälle mit einem geringeren SES wider. Bei mehrfach besetzten Zellen im Fall kategorialer SES-Indikatoren wird der mittlere Anteil von Fällen mit niedrigerem SES eingesetzt.

Alle Analysen wurden mit einem Gewichtungsfaktor durchgeführt, der Abweichungen der Stichprobe von der Bevölkerungsstruktur (Stand 31.12.2010) hinsichtlich Alter, Geschlecht, Region, Staatsangehörigkeit, Gemeindetyp und Bildungsstand des Haushaltsvorstandes (Mikrozensus 2009) korrigiert. Ferner wurde für die ehemaligen Teilnehmerinnen und Teilnehmer der KiGGSBasiserhebung die unterschiedliche Wiederteilnahmebereitschaft mittels Gewichtung nach relevanten Merkmalen aus der KiGGS-Basiserhebung ausgeglichen. Für die Berechnung der Trendanalysen wurden auch die Daten der Basiserhebung bezüglich der oben genannten Merkmale neu gewichtet und auf den Bevölkerungsstand zum 31.12.2010 altersstandardisiert. Details der Methodik von KiGGS Welle 1 sind an anderer Stelle ausführlich beschrieben [23].

Um sowohl die Gewichtung als auch die Korrelation der Teilnehmenden innerhalb einer Gemeinde zu berücksichtigen, wurden die Konfidenzintervalle und p-Werte mit Verfahren für komplexe Stichproben berechnet. Gruppenunterschiede wurden mit dem nach Rao-Scott über die F-Verteilung korrigierten ChiQuadrat-Test für komplexe Stichproben auf Signifikanz geprüft. Unterschiede werden als statistisch signifikant angesehen, wenn sich die Konfidenzintervalle nicht überschneiden bzw. die Irrtumswahrscheinlichkeit (p) einen Wert kleiner als 0,05 annimmt. Alle Analysen wurden mit dem Statistikprogramm Stata 12.1 SE durchgeführt. 


\section{Operationalisierung der statusbildenden Merkmale}

Bei der Überarbeitung der Operationalisierung des sozioökonomischen Status wurde die grundlegende Konzeption des bislang verwendeten WSI-Schichtindex aus der KiGGS-Basiserhebung beibehalten $[10,11]$. Auch der überarbeitete SES-Index basiert auf den 3 Statusdimensionen Bildung, Beruf und Einkommen, die aus den Angaben der Eltern generiert werden. Sie gehen gleichwertig in den als Punktsummenscore berechneten Index ein. Im Unterschied zur Operationalisierung in den Studien GEDA und DEGS werden in der KiGGS-Studie alle Merkmale als Haushaltsmerkmale operationalisiert. Dazu werden die Einzeldimensionen Bildung und Beruf für beide Eltern getrennt erhoben und anschließend nach dem in - Tab. 1 dargestellten Schema mit Punktwerten versehen. Anschließend wird dem Haushalt das Maximum der Elternangaben zugewiesen. In jeder Dimension werden mindestens 1 und höchstens 7 Punkte vergeben, dabei sind auch Zwischenabstufungen möglich. Die Abstände in den Punktwerten spiegeln Differenzen im Hinblick auf externe Kriterien wider. Es kann von einer metrischen Skalierung der Einzeldimensionen ausgegangen werden.

Zur Klassifikation der Bildungsabschlüsse als Haushaltsmerkmal wurden Gruppen auf Basis der internationalen Klassifikation „Comparative Analyses of Social Mobility in Industrial Nations" (CASMIN) verwendet [24]. Die Klassifikation unterscheidet 9 Bildungsgruppen, die sich anhand von Kombinationen aus schulischen und berufsqualifizierenden Abschlüssen ergeben. Die auf den Bereich 1 bis 7 standardisierten Punktwerte spiegeln dabei die Löhne wider, die Personen mit entsprechenden Abschlüssen in Deutschland durchschnittlich erzielen. Für jeden Haushalt wurde das Maximum der verfügbaren Elternangaben zugewiesen.

Um den Berufsstatus zu erfassen, wurden der Berufsstatus der Befragungsperson und der Berufsstatus des Hauptverdieners im Haushalt verglichen und der höhere Wert dem Haushalt zugewiesen. Als Kriterium für die Zuweisung von

Bundesgesundheitsbl 2014 · 57:762-770 DOI 10.1007/s00103-014-1974-8

(c) Springer-Verlag Berlin Heidelberg 2014

T. Lampert · S. Müters · H. Stolzenberg · L. E. Kroll · KiGGS Study Group

Messung des sozioökonomischen Status in der KiGGS-Studie. Erste Folgebefragung (KiGGS Welle 1)

\section{Zusammenfassung}

Der Beitrag beschreibt die Messung des sozioökonomischen Status (SES) in der ersten Folgebefragung der KiGGS-Studie (KiGGS Welle 1), die in den Jahren 2009 bis 2012 durchgeführt wurde. Verwendet wird ein mehrdimensionaler Index, der als Punktsummenscore auf Basis von Angaben der Eltern zu ihrer Schulbildung und beruflichen Qualifikation, zu ihrer beruflichen Stellung und zum Netto-Äquivalenzeinkommen berechnet wird. Gegenüber dem Vorgehen in der KiGGS-Basiserhebung wurden einige Änderungen vorgenommen, wie z. B. die Bevorzugung metrischer statt ordinaler Skalierung, die Verwendung internationaler Kriterien bei der Kategorisierung der Variablen und der Zuweisung von Punktwerten sowie die verteilungsbasierte Abgrenzung der Statusgruppen. Diese Änderungen, die mittlerweile auch in den anderen Komponenten des Gesundheitsmonitorings des Robert
Koch-Instituts, d. h. der Studie "Gesundheit in Deutschland aktuell“ (GEDA) und der "Studie zur Gesundheit Erwachsener in Deutschland" (DEGS1), umgesetzt wurden, sollen die Analyse zeitlicher Entwicklungen und Trends ermöglichen, die internationale Vergleichbarkeit der Daten gewährleisten und den Politikund Praxistransfer der Ergebnisse unterstützen. Um die Verwendung des überarbeiteten Instrumentes zu demonstrieren, werden exemplarisch Ergebnisse der KiGGS Welle 1 zum Zusammenhang zwischen dem soziökonomischen Status und dem allgemeinen Gesundheitszustand von 3- bis 17-jährigen Kindern und Jugendlichen berichtet.

\section{Schlüsselwörter}

Sozioökonomischer Status · Soziale Ungleichheit · Gesundheitliche Ungleichheit . Gesundheitssurvey. Gesundheitsmonitoring

\section{Measurement of socioeconomic status in the KiGGS study. First follow-up (KiGGS Wave 1)}

\section{Abstract}

This article describes the measurement of socioeconomic status in the first follow-up of the KiGGS study (KiGGS Wave 1) conducted from 2009 to 2012. A multidimensional index score was used. The score is the sum of three metric components: Education and Occupational Qualification, Occupational Status, and Net Income. Compared with the approach in the KiGGS baseline study, some modifications were made in accordance with changes in the other components of the health-monitoring program at the Robert Koch Institute, i.e., the German Health Update (GEDA) and the German Health Interview and Examination Survey for Adults (DEGS1). These chang- es facilitate the analysis of temporal developments and trends, ensure international comparability of the data, and support the transfer of the results into politics and practice. In order to demonstrate the application of the revised instrument, we report on exemplary results of KiGGS Wave 1 regarding the relationship between socioeconomic status and the general health status of children and adolescents aged $3-17$ years.

\section{Keywords}

Socioeconomic status - Social inequality . Health inequality $\cdot$ Health survey $\cdot$ Health monitoring
Punktwerten diente der International Socio-Economic-Index of Occupational Status (ISEI) nach Ganzeboom und Treimann [25]. Der ISEI-Index bezieht sich auf berufliche Tätigkeiten, die nach der Berufsklassifikation ISCO-88COM kodiert sind. Die Punktwerte mit dem Variationsbereich von 1 bis 7 wurden auf $\mathrm{Ba}$ sis der GEDA-Studie 2009 generiert [14]. Es wurde das Maximum der Elternangaben zugewiesen.
Beim Einkommen wurde im Einklang mit den Vorgaben der Armuts- und Reichtumsberichterstattung der Bundesregierung und den Empfehlungen für die Berichterstattung zum sozialen Zusammenhalt in Europa das bedarfsgewichtete Haushaltsnettoeinkommen (Netto- ̈̈quivalenzeinkommen) als Indikator verwen$\operatorname{det}[26,27]$. Fehlende Werte beim Haushaltsnettoeinkommen wurden durch ein Regressionsmodell imputiert [14]. Bei 


\begin{tabular}{|c|c|c|c|}
\hline Punkte & $\begin{array}{l}\text { Höchste schulische und berufliche Qualifikation } \\
\text { der Eltern }\end{array}$ & Höchster Berufsstatus der Eltern & $\begin{array}{l}\text { Netto-Äquivalenzeinkom- } \\
\text { men der Eltern }\end{array}$ \\
\hline $1,0-1,9$ & $\begin{array}{l}\text { Kein schulischer und } \\
\text { kein beruflicher Abschluss (1a: 1,0) } \\
\text { Hauptschulabschluss und } \\
\text { kein beruflicher Abschluss (1b: 1,7) }\end{array}$ & $\begin{array}{l}\text { Landwirt: } 10 \text { ha und mehr }(1,0) \\
\text { Landwirt o. n. A. }(1,0) \\
\text { Landwirt: unter } 10 \text { ha }(1,1) \\
\text { Ungelernte Arbeiter }(1,3) \\
\text { Angelernte Arbeiter }(1,8) \\
\text { Arbeiter o. n. A. }(1,9)\end{array}$ & $\begin{array}{l}\leq 664 \operatorname{EUR}(1,0) \\
665-787 \operatorname{EUR}(1,5)\end{array}$ \\
\hline $2,0-2,9$ & $\begin{array}{l}\text { Realschulabschluss oder POS Abschluss und } \\
\text { kein beruflicher Abschluss }(2 \mathrm{~b}: 2,8)\end{array}$ & $\begin{array}{l}\text { Vorarbeiter, Kolonnenführer }(2,0) \\
\text { Gelernte oder Facharbeiter }(2,1) \\
\text { Meister, Polier, Brigadier }(2,4) \\
\text { Angestellte mit ausführender Tätigkeit }(2,4) \\
\text { Mithelfende Familienangehörige }(2,4) \\
\text { Sonstiges o. } n \text {. A. }(2,9) \\
\text { Beamte im einfachen Dienst }(2,9)\end{array}$ & $\begin{array}{l}\text { 788-897 EUR }(2,0) \\
\text { 898-999 EUR }(2,5)\end{array}$ \\
\hline $3,0-3,9$ & $\begin{array}{l}\text { Kein schulischer Abschluss oder Hauptschulab- } \\
\text { schluss und Ausbildung/Lehre/Fachschule (1c: } 3,0) \\
\text { Realschulabschluss, POS und Ausbildung/Lehre/ } \\
\text { Fachschule (2a: } 3,6) \\
\text { FH-Reife, Abitur, EOS und } \\
\text { kein beruflicher Abschluss (2c-gen: } 3.7)\end{array}$ & $\begin{array}{l}\text { Selbstständige: keine Mitarbeiter }(3,5) \\
\text { Angestellte mit qualifizierter Tätigkeit }(3,6) \\
\text { Selbstständige: } 1 \text { bis } 4 \text { Mitarbeiter }(3,6) \\
\text { Angestellte o. } \text {. } \text { A. }(3,7) \\
\text { Selbstständig im Handel, Gewerbe etc. }(3,9)\end{array}$ & $\begin{array}{l}1000-1095 \text { EUR }(3,0) \\
1096-1190 \text { EUR }(3,5)\end{array}$ \\
\hline $4,0-4,9$ & $\begin{array}{l}\text { FH-Reife, Abitur, EOS und Ausbildung/Lehre/Fach- } \\
\text { schule (2c-voc: } 4,8)\end{array}$ & $\begin{array}{l}\text { Selbstständige oder Freiberufler o. n. A. }(4,0) \\
\text { Beamte im mittleren Dienst }(4,1) \\
\text { Angestellte mit verantwortlicher Tätigkeit }(4,2) \\
\text { Selbstständige: } 5 \text { oder mehr Mitarbeiter }(4,2) \\
\text { Selbstständige: PGH-Mitglied }(4,2) \\
\text { Angestellte mit umfassender Führungstätigkeit }(4,7)\end{array}$ & $\begin{array}{l}1191-1293 \text { EUR }(4,0) \\
1294-1400 \text { EUR }(4,5)\end{array}$ \\
\hline $5,0-5,9$ & Kategorie nicht besetzt & $\begin{array}{l}\text { Beamte o. n. A. }(5,0) \\
\text { Beamte im gehobenen Dienst }(5,2) \\
\text { Freiberufler: keine Mitarbeiter }(5,8)\end{array}$ & $\begin{array}{l}1401-1524 \text { EUR }(5,0) \\
1525-1667 \operatorname{EUR}(5,5)\end{array}$ \\
\hline $6,0-7,0$ & $\begin{array}{l}\text { FH-Reife, Abitur, EOS und } \\
\text { Bachelor, Diplom FH (3a: 6,1) } \\
\text { FH-Reife, Abitur, EOS und } \\
\text { Master/Magister/Diplom, Promotion (3b: 7.0) }\end{array}$ & $\begin{array}{l}\text { Akademiker im freien Beruf }(6,2) \\
\text { Beamte im höheren Dienst }(6,4) \\
\text { Freiberufler: } 1 \text { bis } 4 \text { Mitarbeiter }(6,8) \\
\text { Freiberufler: } 5 \text { oder mehr Mitarbeiter }(7,0)\end{array}$ & $\begin{array}{l}1668-1938 \text { EUR }(6,0) \\
1939-2381 \text { EUR }(6,5) \\
\geq 2382 \operatorname{EUR}(7,0)\end{array}$ \\
\hline
\end{tabular}

kategorialen Einkommensangaben wurden die Befragten analog zum Mikrozensus auf das entsprechende Einkommensintervall verteilt [28]. Für die Ermittlung der Punktwerte wurde ausgehend vom Netto-Äquivalenzeinkommen eine verteilungsbasierte Abgrenzung von 13 gleich großen Gruppen vorgenommen, sodass der Abstand zwischen den Einkommensgruppen jeweils einem Punktwert von 0,5 entspricht. Dazu wurden die Angaben zum Alter der Eltern, ihrer Bildung und ihrem Berufsstatus sowie regionalstatistische Informationen des Statistischen Bundesamtes zum mittleren Haushaltsnettoeinkommen der Wohnregion der Befragten verwendet.

\section{Berechnung des mehrdimensionalen SES-Index und Abgrenzung von Statusgruppen}

Der überarbeitete SES-Index wird als Punktsummenscore auf Basis der in den Einzeldimensionen Bildung, Berufsstatus und Einkommen zugewiesenen Punktwerte berechnet. Er ist als Haushaltsmerkmal operationalisiert, Kinder aus demselben Haushalt bekommen folglich den gleichen Wert zugewiesen. Da die 3 Subskalen mit dem gleichen Gewicht in die Berechnung eingehen, kann der SESIndex Werte zwischen 3,0 und 21,0 annehmen. Der SES-Index kann in Analysen als metrische Variable eingehen, oder es kann eine Kategorisierung in mehrere Statusgruppen vorgenommen werden. Die Gruppen spiegeln dann die Rangfol- ge der Kinder mit Blick auf den sozialen Status der Haushalte, in denen sie leben, wider. Dazu wird eine verteilungsbasierte Abgrenzung in 5 gleich stark besetzte Gruppen (Quintile) vorgeschlagen, wobei die 3 mittleren Gruppen (2. bis 4. Quintil) zusammengefasst werden. Diese dreistufige Skala (niedriger, mittlerer und hoher SES) ermöglicht einen Vergleich zwischen den - gemessen an der Kumulation von Bildungsabschlüssen, Berufsstatus und Einkommen - 20\% der Kinder und Jugendlichen, die in sozioökonomisch benachteiligten bzw. begünstigten Verhältnissen aufwachsen, mit einer breit definierten Mitte, die $60 \%$ der Kinder und Jugendlichen umfasst. Die Kategorien, Grenzwerte und der zugehörige Anteil von Teilnehmerinnen und Teilnehmern in KiGGS Welle 1 sind in $\bullet$ Tab. 2 darge- 
Tab. 2 Sozioökonomischer Status der Familien in KiGGS Welle 1 ( $n=12.292)$

\begin{tabular}{lllll}
\hline $\begin{array}{l}\text { Bezeichnung } \\
\text { der Kategorie }\end{array}$ & Quintil des SES & Niedrigster Wert & Höchster Wert & $\begin{array}{l}\text { Anteil gewichtet } \\
\text { (\%) }\end{array}$ \\
\hline "Niedrig" & 1. Quintil & 3,0 & 8,4 & 20,2 \\
\hline "Mittel" & 2. Quintil & 8,5 & 10,5 & 19,7 \\
\cline { 2 - 5 } & 3. Quintil & 10,6 & 12,6 & 19,8 \\
\cline { 2 - 5 } & 4. Quintil & 12,7 & 15,4 & 20,2 \\
\hline "Hoch" & 5. Quintil & 15,5 & 21,0 & 20,1 \\
\hline
\end{tabular}

Tab. 3 Korrelationskoeffizienten für den Zusammenhang zwischen dem SES-Index (Gesamtscore) und den Teildimensionen Bildung, Beruf und Einkommen (Subscores) in KiGGS Welle 1 $(n=12.292)$ und KiGGS-Basiserhebung $(n=17.080)$

\begin{tabular}{|lcccccc}
\hline Korrelationskoeffizienten & (1) & (2) & (3) & (4) & (5) & (6) \\
\hline KiGGS Welle 1 & & & & & & \\
\hline (1) SES-Gesamtscore & 1,00 & & & & & \\
\hline (2) SES-Quintile & 0,96 & 1,00 & & & & \\
\hline (3) SES-Gruppen & 0,89 & 0,89 & 1,00 & & & \\
\hline (4) SES-Subscore Bildung & 0,84 & 0,79 & 0,76 & 1,00 & & \\
\hline (5) SES-Subscore Beruf & 0,79 & 0,74 & 0,71 & 0,58 & 1,00 & \\
\hline (6) SES-Subscore Einkommen & 0,85 & 0,84 & 0,75 & 0,50 & 0,51 & 1,00 \\
\hline KiGGS-Basiserhebung & & & & & & \\
\hline (1) SES-Gesamtscore & 1,00 & & & & & \\
\hline (2) SES-Quintile & 0,96 & 1,00 & & & & \\
\hline (3) SES-Gruppen & 0,89 & 0,89 & 1,00 & & & \\
\hline (4) WSI-Schichtindex & 0,92 & 0,88 & 0,82 & 1,00 & & \\
\hline (5) WSI-Schichten & 0,85 & 0,84 & 0,77 & 0,92 & 1,00 & \\
\hline
\end{tabular}

stellt. Der Anteil an fehlenden Werten beträgt $0,9 \%$.

In - Tab. 3 ist die Korrelation des SESIndex mit den Teildimensionen sowie die Korrelation des Index mit der früheren Operationalisierung nach Winkler und Stolzenberg [14] in der KiGGS-Basiserhebung dargestellt [11]. Die Indexwerte korrelieren in KiGGS Welle 1 mit $r=0,79$ bis $r=0,85$ mit den Einzelindikatoren. Im Vergleich zu den für Erwachsene berichteten Korrelationen zwischen dem Index und dessen Teildimensionen bewegen sich die Werte in einer vergleichbaren Größenordnung [14]. Für die Einschätzung der neuen Operationalisierung des SES ist es von Bedeutung, den Index im Vergleich mit der früheren Operationalisierung des Sozialstatus für die KiGGSBasiserhebung zu vergleichen. Zwischen beiden Operationalisierungen zeigt sich auf Basis der Daten der Basiserhebung eine Korrelation von $r=0,92$ zwischen den Indexscores sowie $r=0,77$ zwischen den kategorialen Indexwerten.

\section{Ergebnisse zum Zusammenhang zwischen dem sozioökonomischen Status und der Einschätzung der Eltern zum allgemeinen Gesundheitszustand ihrer Kinder}

Nach Einschätzung ihrer Eltern haben $51,7 \%$ (95\%-KI: 49,9-53,4) der 3bis 17-jährigen Kinder und Jugendlichen einen sehr guten und weitere $42,0 \%$ $(40,4-43,6)$ einen guten allgemeinen $\mathrm{Ge}$ sundheitszustand. 5,6\% $(5,0-6,7)$ der Kinder und Jugendlichen weisen einen mittelmäßigen und lediglich $0,6 \%(0,4-$ $0,9)$ und $0,1 \%(0,1-0,3)$ einen schlechten bzw. sehr schlechten Gesundheitszustand auf. Dabei sind keine bedeutsamen Unterschiede zwischen Mädchen und Jungen festzustellen (• Abb. 1).

Der Anteil der Kinder und Jugendlichen mit einem nur mittelmäßigen bis sehr schlechten Gesundheitszustand nimmt im Altersgang zu. Während 5,1\% $(4,0-6,6)$ der 3- bis 6-Jährigen und 4,8 \% $(3,6-6,4)$ der 7- bis 10-Jährigen einen mittelmäßigen bis sehr schlechten Gesund- heitszustand haben, trifft dies in der Gruppe der 11- bis 13-Jährigen auf 6,7\% (5,3$8,4)$ und in der Gruppe der 14- bis 17-Jährigen auf 8,6\% (7,2-10,2) zu. Dieser Anstieg im Übergang vom Kindes- zum Jugendalter ist gleichermaßen bei Mädchen und Jungen zu beobachten (• Abb. 2).

Kinder und Jugendliche aus Familien mit niedrigem sozioökonomischem Status weisen häufiger einen mittelmäßigen bis sehr schlechten allgemeinen Gesundheitszustand auf. In der Altersgruppe der 3- bis 10-Jährigen gilt dies für 9,6\% (6,4$14,3)$ der Kinder aus der niedrigen Statusgruppe, während es in der mittleren und hohen Statusgruppe lediglich 4,5\% $(3,7-5,5)$ bzw. $2,2 \%(1,5-3,2)$ sind. Ähnlich ausgeprägte Unterschiede sind bei den 11 - bis 17 -Jährigen mit 11,5\% (8,8$14,9)$ in der niedrigen, $7,2 \%(6,0-8,7)$ in der mittleren und 4,6\% $(3,4-6,2)$ in der hohen Statusgruppe festzustellen. Die statusspezifischen Unterschiede fallen bei Mädchen und Jungen in beiden betrachteten Altersgruppen ähnlich stark aus (• Abb. 3).

Bei statistischer Kontrolle für Alter, Geschlecht und Wohnregion in einem binär logistischen Regressionsmodell ergibt sich für 3- bis 17-jährige Kinder und Jugendliche aus Familien mit niedrigem sozioökonomischem Status ein im Verhältnis zu den Gleichaltrigen aus Familien mit hohem sozioökonomischem Status um etwa das Dreieinhalbfache erhöhte Risiko für einen mittelmäßigen bis sehr schlechten allgemeinen Gesundheitszustand. Für Kinder und Jugendliche aus der mittleren Statusgruppe ist dieses Risiko um das Doppelte gegenüber der sozial bessergestellten Referenzgruppe erhöht (• Tab.4).

Eine nach Alter und Geschlecht differenzierte Betrachtung deutet bei Mädchen wie Jungen sowohl im Kindes- als auch im Jugendalter auf Unterschiede im allgemeinen Gesundheitszustand zuungunsten der niedrigen im Vergleich zur hohen Statusgruppe hin. Statistisch absichern lassen sich diese Unterschiede sowohl bei 3- bis 10-jährigen als auch bei 11- bis 17-jährigen Mädchen und Jungen. Bei Mädchen sind zudem in beiden Altersgruppen statistisch signifikante Unterschiede zwischen der mittleren und hohen Statusgruppe zu erkennen (• Tab. 4). 


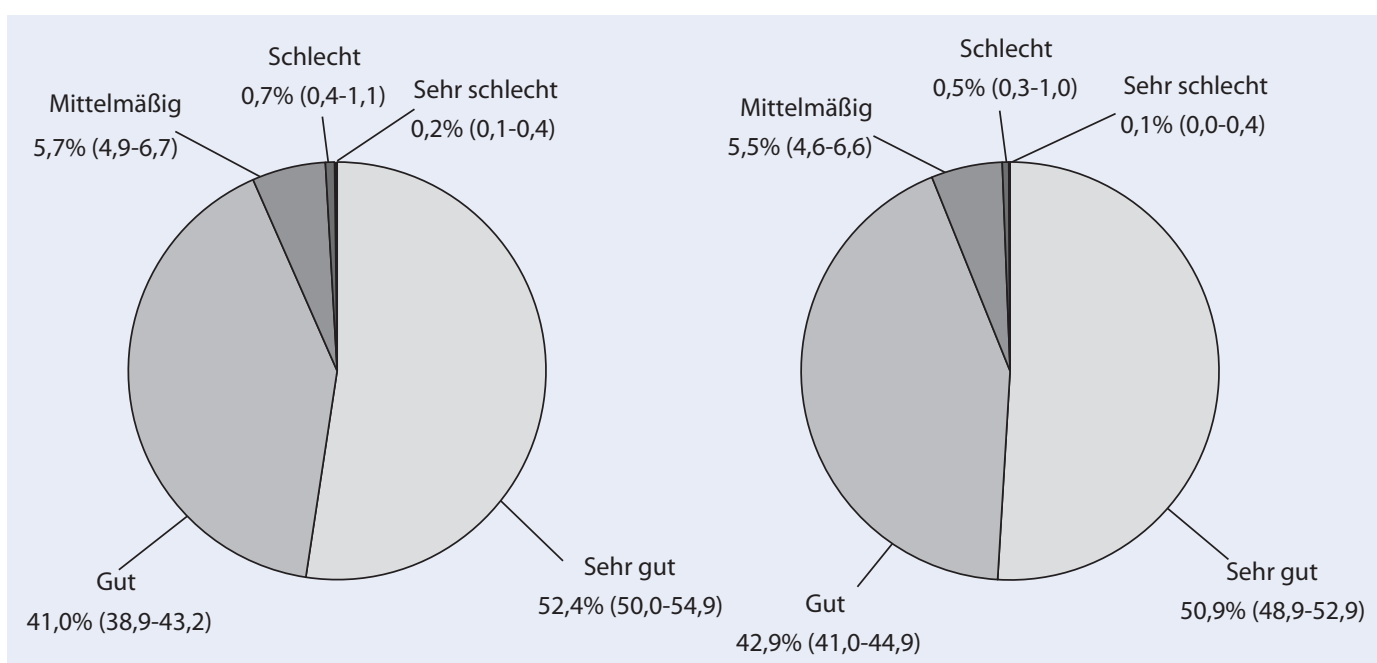

Mädchen
Jungen
Abb. $1 \varangle$ Allgemeiner Gesundheitszustand von 3bis 17-jährigen Mädchen und Jungen $(n=10.961)$
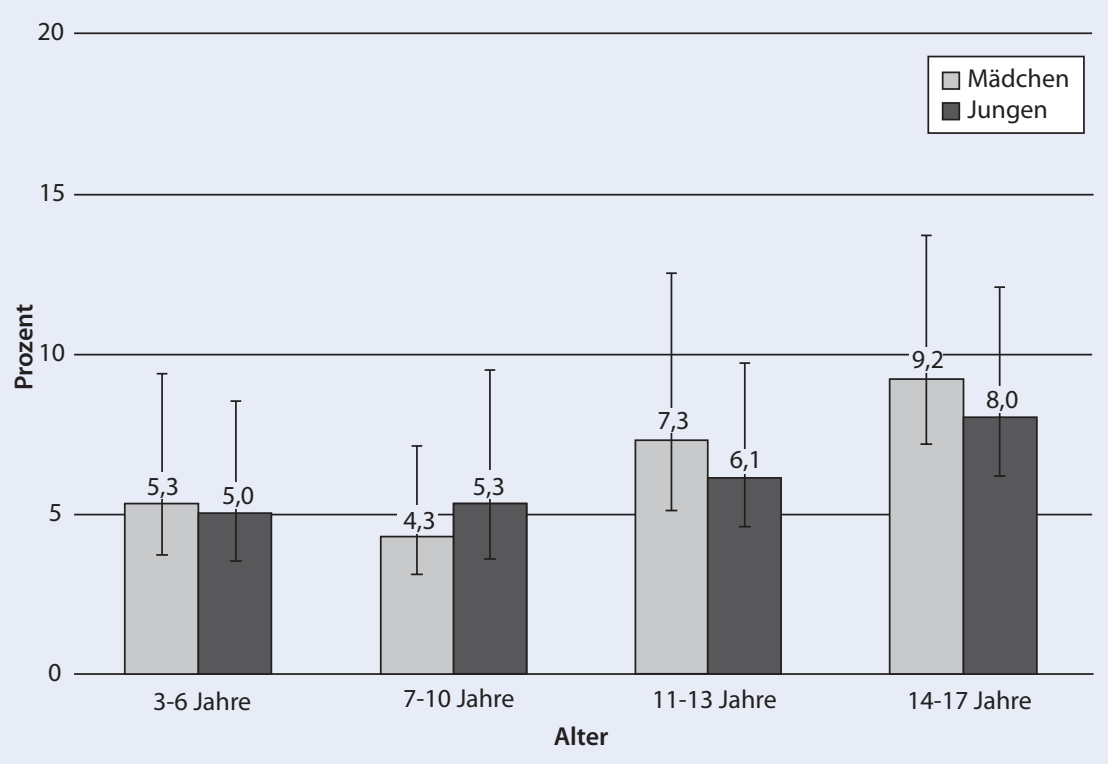

Abb. $2 \Delta$ Allgemeiner Gesundheitszustand (",mittelmäßig“ bis „sehr schlecht") nach Alter und Geschlecht $(n=10.961)$

Auch die Ergebnisse des Poisson-Regressionsmodells, das sich auf den SES-Index unter Berücksichtigung des gesamten Skalenumfangs bezieht, weist auf einen signifikanten Einfluss des sozioökonomischen Status auf den allgemeinen Gesundheitszustand der Kinder und Jugendlichen hin (• Tab. 5). Bei statistischer Kontrolle für Alter, Geschlecht und Wohnregion ergibt sich für Kinder und Jugendliche aus der niedrigen Statusgruppe ein im Vergleich zu den Gleichaltrigen aus der hohen Statusgruppe um den Faktor 1,23 er- höhter Punktwert in Bezug auf den kontinuierlich einbezogenen allgemeinen Gesundheitszustand. Betrachtet man die statusbildenden Merkmale Bildung der Eltern, Berufsstatus der Eltern und NettoÄquivalenzeinkommen des Haushaltes separat, dann erweisen sich diese allesamt als bedeutsam für die Erklärung der Varianz im allgemeinen Gesundheitszustand der Kinder und Jugendlichen. Bei gleichzeitiger Betrachtung der Einzelindikatoren bleiben alle 3 Faktoren statistisch bedeutsam. In den geschlechtsspezifischen
Modellen ist der Einfluss der elterlichen Bildung nicht mehr statistisch bedeutsam, während die Effekte des Berufsstatus der Eltern und der Einkommenssituation der Familie signifikant bleiben.

\section{Diskussion}

Das Gesundheitsmonitoring des RKI macht regelmäßig Daten zur gesundheitlichen Situation von Kindern, Jugendlichen und Erwachsenen für die epidemiologische Forschung und die Gesundheitsberichterstattung verfügbar. Analysen zum Zusammenhang zwischen dem sozioökonomischen Status und dem Gesundheitszustand, dem Gesundheitsverhalten sowie der Gesundheitsversorgung stellen dabei einen Schwerpunkt dar. Um den Anforderungen an ein Gesundheitsmonitoring gerecht werden zu können, wurde der bislang in den Gesundheitssurveys des RKI verwendete WSI-Schichtindex einer kritischen Überprüfung unterzogen und weiterentwickelt. $\mathrm{Zu}$ diesen Anforderungen zählen vor allem die Analyse zeitlicher Entwicklungen und Trends, die internationale Vergleichbarkeit der Daten sowie der Politik- und Praxistransfer der Ergebnisse.

Der überarbeitete Index, der als Index des sozioökonomischen Status, kurz SESIndex, bezeichnet wird, basiert wie der WSI-Schichtindex auf Angaben zu den berufsnahen Dimensionen Bildung, Beruf und Einkommen. Die wichtigsten Unter- 
25

$\square$ Niedrig $\square$ Mittel $\square$ Hoch

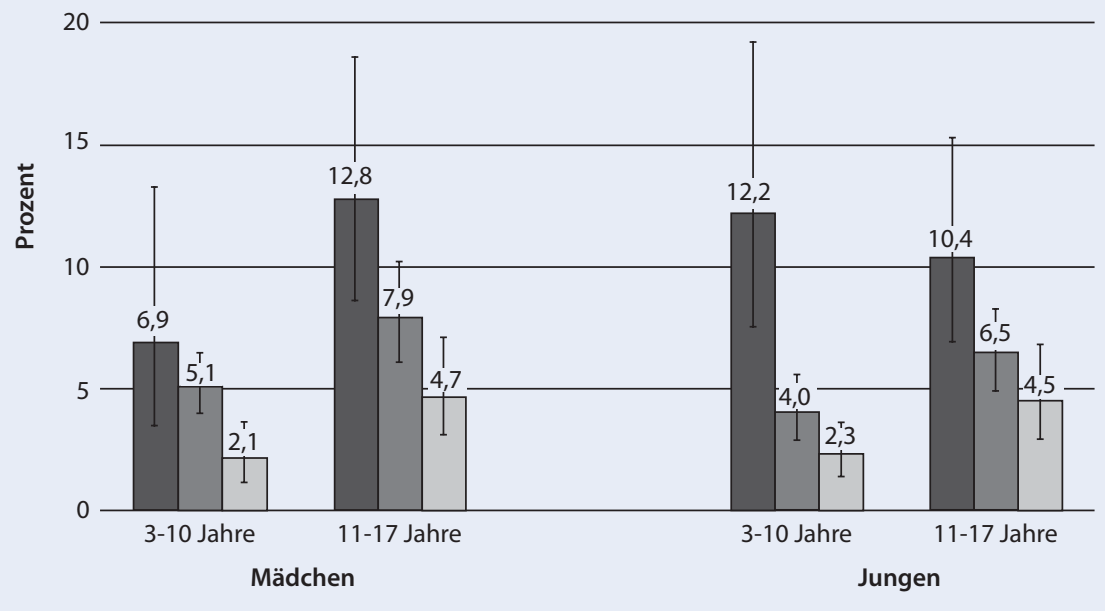

Abb. $3 \Delta$ Allgemeiner Gesundheitszustand ("mittelmäßig" bis „sehr schlecht") nach sozioökonomischem Status und Alter $(n=10.346)$

schiede gegenüber dem bisherigen Vorgehen bestehen in der Operationalisierung der statusbildenden Merkmale und in der Abgrenzung der Statusgruppen auf Basis des als Punktsummenscore ermittelten Index. Während nach dem Operationalisierungsvorschlag von Winkler und Stolzenberg die Ausgangsvariablen Bildung, Beruf und Einkommen in ordinale Skalen überführt werden, erfolgt für den überarbeiteten Index eine metrische Skalierung. Dadurch ergibt sich ein Informationsgewinn, der mit Vorteilen bei der Berechnung des SES-Index und der verteilungsbasierten Abgrenzung von Statusgruppen verbunden ist. Bei der Kategorisierung der Variablen und der Zuweisung von Punktwerten zu den einzelnen Kategorien wurde auf international bewährte Instrumente und Kriterien zurückgegriffen, um die Vergleichbarkeit mit Untersuchungen in anderen Ländern zu erhöhen. Für die Bildung wurde die CASMIN-Klassifikation herangezogen, die auf Angaben zur Schulbildung und beruflichen Qualifikation basiert und für internationale Vergleiche entwickelt wurde [24]. Die Kategorisierung der Angaben zum Beruf bezieht sich auf den ISEI-Index als einem der wichtigsten internationalen Maße zur Bestimmung des beruflichen Status [25]. Durch die Einbeziehung des Netto-Äquivalenzeinkommens anstelle des Haushaltsnettoeinkommens wurde der auf na- tionaler und internationaler Ebene ausgesprochenen Empfehlung Rechnung getragen, bei Aussagen zur Einkommenssituation des Haushaltes auch die Haushaltsgröße und die Haushaltszusammensetzung zu berücksichtigen [26].

Ein weiterer Vorteil des überarbeiteten SES-Index ist in der verteilungsbasierten Abgrenzung der Statusgruppen zu sehen. Für das Gesundheitsmonitoring wird ausgehend von einer Quintilbildung ein dreistufiges Konzept vorgeschlagen, wobei die niedrige und die hohe Statusgruppe jeweils $20 \%$ (1. bzw. 5. Quintil) und die mittlere Statusgruppe $60 \%$ der Bevölkerung umfassen. Denkbar sind aber ebenso, je nach Fragestellung und Erkenntnisinteresse, eine Betrachtung von 5 Statusgruppen (1. bis 5. Quintil) oder Betrachtungen anhand anderer Einteilungen, wie z. B. Terzilen oder Quartilen. Durch die verteilungsbasierte Abgrenzung von Statusgruppen wird das zugrunde liegende Konzept relativer sozialer und gesundheitlicher Ungleichheit unterstrichen, das davon ausgeht, dass die Zugehörigkeit zu den sozioökonomisch am schlechtesten bzw. am besten gestellten Gruppen auch dann für die Gesundheit relevant ist, wenn sich der allgemeine Wohlstand vergrößert und die allgemeinen Lebensbedingungen verbessern. Für die Analyse zeitlicher Entwicklungen und Trends bedeutet dies, dass zu jedem Zeitpunkt die jeweils $20 \%$ der Bevölkerung mit der größten sozioökonomischen Benachteiligung mit den $20 \%$ der Bevölkerung mit der größten sozioökonomischen Begünstigung verglichen werden, und zwar unabhängig vom jeweiligen allgemeinen Wohlstandsniveau.

Die vorgestellten empirischen Ergebnisse für den allgemeinen Gesundheitszustand veranschaulichen, dass der überarbeitete SES-Index sehr gut diskriminiert. Sowohl das Muster als auch die Stärke der Zusammenhänge stimmen mit den in anderen Studien berichteten Ergebnissen überein. Auch die alters- und geschlechtsspezifischen Variationen der Zusammenhänge stehen im Einklang mit vergleichbaren Forschungsergebnissen, z. B. denen der Studie „Health Behaviour in Schoolaged Children" (HBSC) [4, 29].

Ein mehrdimensionaler SES-Index ist geeignet, um das Ausmaß und die Entwicklung der gesundheitlichen Ungleichheit zu beschreiben. Ebenso kann er in epidemiologischen Studien z. B. zur Ermittlung von Risikoprofilen für bestimmte Erkrankungen herangezogen werden, und zwar sowohl als eigenständige Einflussgröße als auch zur Confounder-Kontrolle $[14,15]$. $\mathrm{Zu}$ berücksichtigen ist allerdings, dass der Aussagekraft der mit einem mehrdimensionalen Index erzielten Ergebnisse Grenzen gesetzt sind. Dies wird besonders deutlich, wenn nach Erklärungszusammenhängen für die beobachteten sozioökonomischen Unterschiede im Gesundheitszustand gefragt wird oder konkrete Zielgruppen für Interventionen identifiziert werden sollen. Analysen mit den Einzelindikatoren Bildung, Beruf und Einkommen, wie sie auch in diesem Beitrag vorgenommen wurden, sind hier aufschlussreicher, da sie eher Rückschlüsse auf die Bedeutung von z. B. materiellen Lebensbedingungen, sozialen Teilhabechancen oder gesundheitsrelevanten Einstellungen und Verhaltensweisen erlauben $[27,30]$. Häufig wird es aber darüber hinaus vertiefender Analysen zur sozialen Lage bzw. Lebenslage der Kinder und Jugendlichen unter Berücksichtigung weiterer, durch den sozioökonomischen Status nicht direkt erfasster Merkmale, wie z. B. Erwerbstatus der Eltern, Familienkomposition oder Migrationshintergrund der Familie, bedürfen. 
Tab. 4 Einfluss des sozioökonomischen Status (SES) auf den allgemeinen Gesundheitszustand (",mittelmäßig“ bis ,sehr schlecht") von 3- bis 17-jährigen Kindern und Jugendlichen. Ergebnisse binär logistischer Regressionen bei Kontrolle für Alter und Wohnregion, im Gesamtmodell zusätzlich für Geschlecht (OR mit 95\%-KI und p-Wert, $n=10.346$ )

\begin{tabular}{|llll}
\hline & \multicolumn{1}{l}{ SES } & \\
& Niedrig & Mittel & Hoch \\
& OR $(95-K I)$ p-Wert & OR $(95-K I)$ p-Wert & \\
\hline Mädchen & & & \\
\hline 3 bis 10 Jahre & $3,62(1,44-9,08) 0,01$ & $2,53(1,36-4,72) 0,00$ & Ref. \\
\hline 11 bis 17 Jahre & $2,99(1,59-5,63) 0,00$ & $1,76(1,04-2,99) 0,04$ & Ref. \\
\hline Gesamt & $3,40(2,00-5,77) 0,00$ & $2,12(1,45-3,08) 0,00$ & Ref. \\
\hline Jungen & & & \\
\hline 3 bis 10 Jahre & $5,87(2,75-12,53) 0,00$ & $1,77(0,94-3,32) 0,08$ & Ref. \\
\hline 11 bis 17 Jahre & $2,44(1,27-4,67) 0,01$ & $1,44(0,84-2,48) 0,18$ & Ref. \\
\hline Gesamt & $3,72(2,23-6,20) 0,00$ & $1,62(1,09-2,40) 0,02$ & Ref. \\
\hline Gesamt & & & \\
\hline 3 bis 10 Jahre & $4,84(2,64-8,90) 0,00$ & $2,12(1,36-3,31) 0,00$ & Ref. \\
\hline 11 bis 17 Jahre & $2,70(1,70-4,30) 0,00$ & $1,60(1,08-2,38) 0,02$ & Ref. \\
\hline Gesamt & $3,60(2,46-5,27) 0,00$ & $1,87(1,41-2,50) 0,00$ & Ref. \\
\hline
\end{tabular}

Tab. 5 Einfluss des sozioökonomischen Status (SES) und der SES-Einzelindikatoren Bildung, Beruf und Einkommen auf den allgemeinen Gesundheitszustand von 3- bis 17-jährigen Kindern und Jugendlichen. Ergebnisse von Poisson-Regressionsmodellen für den gesamten Skalenumfang bei getrennter und gleichzeitiger Betrachtung der Einflussfaktoren und Kontrolle für Alter und Wohnregion, im Gesamtmodell auch für Geschlecht (RII mit $95 \%$-KI und p-Wert, $n=10.309)$

\begin{tabular}{|lll}
\hline & $\begin{array}{l}\text { Getrennte Betrachtung } \\
\text { RII }(95 \%-K I) \text { p-Wert }\end{array}$ & $\begin{array}{l}\text { Gleichzeitige Betrachtung } \\
\text { RII }(95 \%-K I) \text { p-Wert }\end{array}$ \\
\hline Mädchen & & \\
\hline SES-Gesamtscore & $1,22(1,15-1,31) 0,00$ & $1,07(0,97-1,17) 0,17$ \\
\hline Bildung & $1,19(1,10-1,28) 0,00$ & $1,11(1,02-1,20) 0,01$ \\
\hline Beruf & $1,20(1,12-1,28) 0,00$ & $1,09(1,02-1,16) 0,01$ \\
\hline Einkommen & $1,19(1,12-1,26) 0,00$ & \\
\hline Jungen & & \\
\hline SES-Gesamtscore & $1,24(1,17-1,32) 0,00$ & $1,07(0,99-1,15) 0,09$ \\
\hline Bildung & $1,20(1,12-1,28) 0,00$ & $1,11(1,03-1,20) 0,01$ \\
\hline Beruf & $1,22(1,15-1,30) 0,00$ & $1,11(1,03-1,19) 0,00$ \\
\hline Einkommen & $1,21(1,14-1,29) 0,00$ & \\
\hline Gesamt & & \\
\hline SES-Gesamtscore & $1,23(1,18-1,29) 0,00$ & $1,07(1,00-1,14) 0,04$ \\
\hline Bildung & $1,19(1,13-1,25) 0,00$ & $1,11(1,04-1,18) 0,00$ \\
\hline Beruf & $1,21(1,15-1,26) 0,00$ & $1,10(1,04-1,16) 0,00$ \\
\hline Einkommen & $1,20(1,15-1,25) 0,00$ &
\end{tabular}

Ebenso ist der Blick auf die Kontexte zu richten, in denen sich das Leben der Heranwachsenden abspielt, neben der Familie z. B. Kindertagesstätte, Schule, Vereine und Nachbarschaft, um zu verstehen, warum die gesundheitliche Entwicklung im Kindes- und Jugendalter in engem Zusammenhang mit dem sozioökonomischen Status steht $[5,10]$.

\section{Einhaltung ethischer Richtlinien}

Interessenkonflikt. T. Lampert, S. Müters, H. Stolzenberg und L. E. Kroll geben an, dass kein Interessenkonflikt besteht.

Finanzierung der Studie. Die Studie wurde mit Mitteln des Robert Koch-Instituts und des Bundesministeriums für Gesundheit finanziert.

\section{Literatur}

1. West $P$ (1997) Health inequalities in the early years. Is there equalization in youth? Soc Sci Med 44:833-858

2. Starfield B, Riley AW, Witt WP, Robertson J (2002) Social class gradients in health during adolescence. J Epidemiol Community Health 56:354-361

3. Chen E, Martin AD, Matthews KA (2006) Socioeconomic status and health: do the gradients differ within childhood and adolescence? Soc Sci Med 62:2161-2170

4. Richter M (2005) Gesundheit und Gesundheitsverhalten im Jugendalter - Der Einfluss sozialer Ungleichheit. VS Verlag für Sozialwissenschaften, Wiesbaden

5. Lampert T, Richter M (2009) Gesundheitliche Ungleichheit bei Kindern und Jugendlichen. In: Richter M, Hurrelmann K (Hrsg) Gesundheitliche Ungleichheit - Theorien, Konzepte und Methoden, 2., aktualisierte Aufl. VS Verlag für Sozialwissenschaften, Wiesbaden, S 209-230

6. Dragano N, Lampert T, Siegrist J (2010) Wie baut sich soziale und gesundheitliche Ungleichheit im Lebenslauf auf? In: Deutsches Jugendinstitut (Hrsg) Mehr Chancen für gesundes Aufwachsen. Materialien zum 13. Kinder- und Jugendbericht. Verlag Deutsches Jugendinstitut, München, S 1350

7. Lampert T, Kurth B-M (2007) Sozialer Status und Gesundheit von Kindern und Jugendlichen - Ergebnisse der KiGGS-Studie. Deutsches Ärzteblatt 104:2944-2949

8. Lampert T, Mensink GBM, Kleiser C et al (2009) Entwicklung und Evaluation der nationalen Gesundheitsziele für Kinder und Jugendliche? Welchen Beitrag leistet der Kinder- und Jugendgesundheitssurvey des Robert Koch-Instituts (KiGGS)? Bundesgesundheitsbl Gesundheitsforsch Gesundheitsschutz 52:905-918

9. Lampert T (2011) Soziale Ungleichheit und Gesundheit im Kindes- und Jugendalter. Pädiatrie up2date 6:117-142

10. Lampert T, Schenk L, Stolzenberg H (2002) Konzeptualisierung und Operationalisierung sozialer Ungleichheit im Kinder- und Jugendgesundheitssurvey. Gesundheitswesen 64:48-52

11. Lange $M$, Kamtsiuris $P$, Lange $C$ et al (2007) Messung soziodemographischer Merkmale im Kinderund Jugendgesundheitssurvey (KiGGS) und ihre Bedeutung am Beispiel der Einschätzung des allgemeinen Gesundheitszustands. Bundesgesundheitsbl Gesundheitsforsch Gesundheitsschutz 50:578-589

12. Winkler J, Stolzenberg H (1999) Der Sozialschichtindex im Bundes-Gesundheitssurvey. Gesundheitswesen 61:178-183

13. Kurth BM, Lange C, Kamtsiuris $P$, Hölling H (2009) Gesundheitsmonitoring am Robert Koch-Institut, Sachstand und Perspektiven. Bundesgesundheitsbl Gesundheitsforsch Gesundheitsschutz 52:557-570 
14. Lampert T, Kroll LE, Müters S et al (2013) Messung des sozioökonomischen Status in der Studie „, Gesundheit in Deutschland aktuell" (GEDA). Bundesgesundheitsbl Gesundheitsforsch Gesundheitsschutz 56:131-143

15. Lampert T, Kroll LE, Müters S et al (2013) Messung des sozioökonomischen Status in der Studie zur Gesundheit Erwachsener in Deutschland (DEGS 1). Bundesgesundheitsbl Gesundheitsforsch Gesundheitsschutz 56:631-636

16. Kamtsiuris $P$, Lange $M$, Schaffrath Rosario A (2007) Der Kinder- und Jugendgesundheitssurvey (KiGGS): Stichprobendesign, Response und Nonresponse-Analyse. Bundesgesundheitsbl Gesundheitsforsch Gesundheitsschutz 50:547-556

17. Kurth BM, Kamtsiuris $P$, Hölling $H$, Schlaud $M$, Dölle R, Ellert U, Kahl H, Knopf H, Lange M, Mensink GBM, Neuhauser $H$, Schaffrath Rosario A, Scheidt-Nave C, Schenk L, Schlack R, Stolzenberg $\mathrm{H}$, Thamm M, Thierfelder W, Wolf U (2008) The challenge of comprehensively mapping children's health in a nation-wide health survey: design of the German KiGGS Study. BMC Public Health 8:196

18. Hölling $H$, Schlack R, Kamtsiuris $P$, Butschalowsky H, Schlaud M, Kurth BM (2012) Die KiGGS-Studie. Bundesweit repräsentative Längs- und Querschnittstudie zur Gesundheit von Kindern und Jugendlichen im Rahmen des Gesundheitsmonitorings am Robert Koch-Institut. Bundesgesundheitsbl Gesundheitsforsch Gesundheitsschutz 55:836-842

19. DeBruin A, Picavet HSJ, Nossikov A (1996) Health interview surveys. Towards harmonization of methods and instruments. WHO regional publications. Euopean Series No 58:51-53

20. Mackenbach J, Kunst A (1997) Measuring the magnitude of socio-economic inequalities in health: an overview of available measures illustrated with two examples from Europe. Soc Sci Med 44:757-771

21. Batty D, Der G, Macintyre S, Deary I (2006) Does IQ explain socioeconomic inequalities in health? Evidence from a population based cohort study in the west of Scotland. BMJ 332:580-584

22. Mackenbach JP, Stirbu I, Roskam AJ et al (2008) Socioeconomic inequalities in health in 22 European countries. N Engl J Med 358:2468-2481

23. Lange M, Butschalowsky HG, Jentsch F, Kuhnert $R$, Schaffrath Rosario A, Schlaud M, Kamtsuris P, KiGGS Study Group (2014) Die erste KiGGS-Folgebefragung (KiGGS Welle 1). Studiendurchführung, Stichprobendesign und Response. Bundesgesundheitsbl Gesundheitsforsch Gesundheitschutz 57. doi: 10.1007/s00103-014-1973-9

24. Brauns H, Scherer S, Steinmann S (2003) The CASMIN Educational Classification in International Comparative Research. In: Hoffmeyer-Zlotnik JHP, Wolf C (Hrsg) Advances in cross-national comparison. Kluwer, New York, S 221-244

25. Ganzeboom HGB, Treimann DJ (2003) Three internationally standardized measures for comparative research on occupational status. In: HoffmeyerZlotnik JHP, Wolf C (Hrsg) Advances in cross-national comparison. Kluwer, New York, S 159-193

26. BMAS (2013) Lebenslagen in Deutschland. Der 4. Armuts- und Reichtumsbericht der Bundesregierung. Bundesministerium für Arbeit und Soziales, Berlin

27. Lampert T, Kroll LE (2009) Messung des sozioökonomischen Status in sozialepidemiologischen Studien. In: Richter M, Hurrelmann K (Hrsg) Gesundheitliche Ungleichheit - Theorien, Konzepte und Methoden, 2., aktualisierte Auflage. VS Verlag für Sozialwissenschaften, Wiesbaden, S 309-334
28. Stauder J, Hüning W (2004) Die Messung von Äquivalenzeinkommen und Armutsquoten auf der Basis des Mikrozensus. In: Landesamt für Datenverarbeitung und Statistik Nordrhein-Westfalen (Hrsg) Statistische Analysen und Studien NRW, Band 13. Landesamt für Datenverarbeitung und Statistik NRW, Düsseldorf, S 9-31

29. Moor I, Pförtner TK, Lampert T, Ravens-Sieberer U, Richter M (2012) Gesundheitliche Ungleichheiten im Jugendalter: Eine Trendanalyse anhand der HBSC-Studie von 2002-2010. Gesundheitswesen 74:S49-S55

30. Jöckel K-H, Babitsch B, Bellach B-M et al (1998) Messung und Quantifizierung soziodemographischer Merkmale in epidemiologischen Studien. In: Ahrens W, Bellach BM, Jöckel KH (Hrsg) Messung sozidemographischer Merkmale in der Epidemiologie. RKI-Schriften 1/1998. MMV Medizin, München, S 7-38 